UB-HET-02-08

November 2002

\title{
Electroweak Radiative Corrections to Weak Boson Production at Hadron Colliders
}

\author{
U. Baur and D. Wackeroth ${ }^{\mathrm{a}}$ \\ ${ }^{\text {a Physics Department }}$ \\ State University of New York at Buffalo \\ Buffalo, NY 14260, USA
}

\begin{abstract}
We summarize the status of calculations of the electroweak radiative corrections to $\mathrm{W}$ and $\mathrm{Z}$ boson production via the Drell-Yan mechanism at hadron colliders. To fully exploit the precision physics potential of the highluminosity environment of the Fermilab Tevatron $p \bar{p}$ (Run II) and the CERN LHC $p p$ colliders, it is crucial that the theoretical predictions are well under control. The envisioned precision physics program includes a precise measurement of the $\mathrm{W}$ boson mass and width, and the (leptonic) weak mixing angle, as well as probing the Standard Model (SM) of electroweak interactions at the highest accessible center-of-mass energies. Some numerical results are presented.
\end{abstract}

\section{Introduction}

The Standard Model of electroweak interactions (SM) so far withstood all experimental challenges and is tested as a quantum field theory at the $0.1 \%$ level [ [1]. However, the mechanism of mass generation in the SM predicts the existence of a Higgs boson which, so far, has eluded direct observation. Direct searches at LEP2 give a $95 \%$ confidence-level lower bound on the mass of the SM Higgs boson of $M_{H}>114.4 \mathrm{GeV}$ [ 2]. Indirect information on the mass of the Higgs boson can be extracted from the $M_{H}$ dependence of radiative corrections to the $\mathrm{W}$ boson mass. With the present knowledge of the $\mathrm{W}$ boson and top quark masses, and the electromagnetic coupling constant, $\alpha\left(M_{Z}^{2}\right)$, the SM Higgs boson mass can be indirectly constrained to $M_{H}=88_{-35}^{+53} \mathrm{GeV}$ [ 11 by a global fit to all electroweak precision data. Future more precise measurements of the $\mathrm{W}$ boson and top quark masses are expected to considerably improve the present indirect bound on $M_{H}$ : with a precision of $27 \mathrm{MeV}(16 \mathrm{MeV})$ for the W boson mass, $M_{W}$, and $2.7 \mathrm{GeV}(1.4 \mathrm{GeV})$ for the top quark mass which are target values for Run IIa (Run IIb) of the Tevatron [ 3], $M_{H}$ can be predicted with an uncertainty of about $35 \%$ $(25 \%)$. In addition, the confrontation of a precisely measured $\mathrm{W}$ boson mass with the indirect SM prediction from a global fit to all electroweak precision data, $M_{W}=80.398 \pm 0.019 \mathrm{GeV}$ [ []], together with a precise measurement of the W width, $\Gamma_{W}$, from the high transverse mass tail, will provide a stringent test of the SM. A detailed discussion of the prospects for the precision measurement of $M_{W}$ and $\Gamma_{W}$, and of the (leptonic) effective weak mixing angle, $\sin ^{2} \theta_{\text {eff }}^{l}$, at Run II and the LHC is given in Refs. [4] and [5], respectively.

In order to measure $M_{W}$ with high precision in a hadron collider environment it is necessary to fully control higher order QCD and electroweak radiative corrections to the $\mathrm{W}$ and $\mathrm{Z}$ production processes. The status of the QCD corrections to $\mathrm{W}$ and $\mathrm{Z}$ boson production at hadron colliders is reviewed in Refs. [6, 77. Here we discuss the electroweak $\mathcal{O}(\alpha)$ corrections to $p \stackrel{(-)}{p} \rightarrow W^{ \pm} \rightarrow l^{ \pm} \nu_{l}$ and $p \stackrel{(-)}{p} \rightarrow \gamma^{*}, Z \rightarrow l^{+} l^{-}(l=e, \mu)$ as presented in detail in Refs. [ 8,9$]$ and [10, 11, 12, 13], concentrating on photon radiation effects and the impact of non-resonant weak corrections on the $\mathrm{W}$ 
width measurement.

\section{Photon Radiation Effects}

We illustrate the effects caused by photon radiation using $\mathrm{Z}$ boson production as an example. The electroweak $\mathcal{O}(\alpha)$ corrections to neutralcurrent Drell-Yan processes naturally decompose into QED and weak contributions, i.e. they build gauge invariant subsets and thus can be discussed separately. The radiation of collinear photons off quarks requires the factorization of the arising mass singularities into the PDFs which introduces a dependence on the QED factorization scale, $\mu_{Q E D}$.

In Fig. 1 we show the effect of the QED corrections on the invariant mass distribution of the final state lepton pair. Photon radiation strongly affects the shape of the $m\left(l^{+} l^{-}\right)$distribution. This is due to the mass singular terms associated with final state photon radiation, which are proportional to $\log \left(\hat{s} / m_{l}^{2}\right)$, where $m_{l}$ is the mass of the final state lepton. At the $\mathrm{Z}$ pole, the differential cross section is reduced by about $30 \%$ in the electron case and by about $20 \%$ in the muon case. Below the Z peak, QED corrections enhance the cross section by up to a factor 2.7 (1.9) for electrons (muons).

When lepton identification requirements are taken into account, the large contributions from mass singular logarithms largely cancel in the electron case because electron and photon momenta are combined for small opening angles between the two particles. For muons, the energy of a photon which traverses the same calorimeter cell as the muon is required to be $E_{\gamma}<2 \mathrm{GeV}$, and only the momenta of muons and photons with $E_{\gamma}<2 \mathrm{GeV}$ are combined. As a result, the mass singular logarithmic terms survive, and the effect of radiative corrections becomes more pronounced, especially at large di-lepton masses.

The distortion of the shape of the Breit-Wigner resonance curve of the $\mathrm{Z}$ boson due to final state photon radiation leads to a shift in the $\mathrm{Z}$ boson mass extracted from data. In the electron channel, the shift is about $100 \mathrm{MeV}$, whereas for $Z \rightarrow \mu^{+} \mu^{-}$one finds approximately $300 \mathrm{MeV}$.

Since the neutrino in $\mathrm{W}$ decays is not observed, it is impossible to reconstruct the $l \nu_{l}$ invariant mass, and the transverse mass $\left(M_{T}\right)$ distribution is frequently used to extract the mass and width of the $\mathrm{W}$ boson. Final state photon radiation in $W \rightarrow l \nu_{l}$ affects the shape of the $M_{T}$ distribution in a similar way as the di-lepton invariant mass distribution in $Z \rightarrow l^{+} l^{-}$. The ratio of the $\mathcal{O}\left(\alpha^{3}\right)$ and lowest order differential cross section as a function of $M_{T}(l \nu)$ with and without lepton identification requirements taken into account is shown in Fig. 2. Since neutrinos do not interact with photons, the shift in $M_{W}$ due to final state photon radiation is about a factor two smaller than in the $\mathrm{Z}$ case. For more details we refer the reader to Ref. [ 9 ].

\section{Non-resonant Weak Corrections to W Production and $\mathrm{W}$ width Measurement}

The virtual radiative corrections to $p \stackrel{(-)}{p} \rightarrow$ $W^{ \pm} \rightarrow l^{ \pm} \nu$ comprise resonant and non-resonant contributions. The non-resonant contributions, which include those originating from the $W Z$ box diagrams, vanish for $\hat{s}=M_{W}^{2}$, and thus were not included in Ref. [ 9]. For precision physics away from the $\mathrm{W}$ pole, however, the non-resonant corrections must be included. These corrections become important at large values of $M_{T}$ due to the presence of large Sudakov-like electroweak logarithms of the form $\log ^{2}\left(M_{T} / M_{W}\right)$, which eventually may be resummed [14].

In Fig. 3 we show the $M_{T}$ distribution including the full $\mathcal{O}(\alpha)$ corrections normalized to the differential cross section in the effective Born approximation (EBA) at the Tevatron for electron and muon final states. Separation cuts and lepton identification requirements to simulate the detector acceptance as described in Ref. [ [9] are taken into account in Fig. 3. For comparison the relative corrections including the resonant $\mathcal{O}(\alpha)$ electroweak corrections only are also shown (dashed line). As expected from the presence of large electroweak Sudakov-like logarithms, the non-resonant weak corrections strongly increase in magnitude with increasing transverse masses. Both, the resonant and non-resonant electroweak corrections reduce the differential cross section.

The high transverse mass tail can be used for a 
direct measurement of the width of the $\mathrm{W}$ boson, $\Gamma_{W}$, which in the SM is predicted to be $\Gamma_{W}(S M)=2.072 \mathrm{GeV}$. In Run I, $\Gamma_{W}$ has been been measured by CDF and D $\varnothing$ with a combined uncertainty of $105 \mathrm{MeV}$ from the $M_{T}$ tail [15. In Run IIa, with an integrated luminosity of $2 \mathrm{fb}^{-1}$, one expects to achieve a precision of $50 \mathrm{MeV}$ per lepton channel and experiment [ [4]. In order to determine how non-resonant electroweak corrections affect the $\mathrm{W}$ width extracted from the tail of the transverse mass distribution, it is useful to consider the ratio of the normalized $M_{T}$ distribution for an arbitrary $\mathrm{W}$ width to the normalized $M_{T}$ distribution with SM W width,

$\mathcal{R}\left(\Gamma_{W}\right)=\frac{\left[d \sigma\left(\Gamma_{W}\right) / d M_{T}\right]}{\left[d \sigma(S M) / d M_{T}\right]} \frac{\sigma(S M)}{\sigma\left(\Gamma_{W}\right)}$

when resonant $\mathcal{O}(\alpha)$ corrections only are taken into account. It is easy to show that $\mathcal{R}\left(\Gamma_{W}\right)$ is proportional to $\Gamma_{W} / \Gamma_{W}(S M)$ for $M_{T} \gg M_{W}$. $\mathcal{R}\left(\Gamma_{W}\right)$ is shown for $p \bar{p} \rightarrow e^{+} \nu_{e}(\gamma)$ at the Tevatron in Fig. A. The dashed, dotted and dashdotted curves represent the predictions for $\Gamma_{W}=$ $2.032 \mathrm{GeV}, 2.042 \mathrm{GeV}$ and $2.052 \mathrm{GeV}$, respectively. The solid line shows the result if, instead of a non-standard $\mathrm{W}$ width and resonant corrections, the full $\mathcal{O}(\alpha)$ electroweak corrections with $\Gamma_{W}(S M)=2.072 \mathrm{GeV}$ are used. The error bars represent the expected statistical uncertainties for $2 \mathrm{fb}^{-1}$. Experimental lepton identification criteria and detector resolution effects are taken into account in Fig. 4.

Figure 4 shows that both, non-resonant electroweak corrections and a $\mathrm{W}$ width smaller than that predicted in the SM, lead to similar effects in the tail of the $M_{T}$ distribution. This effect sets on gradually in case of the non-resonant corrections. For a non-standard W width in contrast, the ratio drops rapidly above the $\mathrm{W}$ pole position, and becomes constant for $M_{T}>110 \mathrm{GeV}$. Since the experimental precision in the tail of the $M_{T}$ distribution quickly decreases with increasing values of the transverse mass, one concludes that nonresonant electroweak corrections, if ignored in the experimental determination of the $\mathrm{W}$ width from the high $M_{T}$ tail, would shift the extracted value of $\Gamma_{W}$ by about $-10 \mathrm{MeV}$.

\section{Conclusions}

Calculations of the full $\mathcal{O}(\alpha)$ electroweak corrections to $\mathrm{W}$ and $\mathrm{Z}$ boson production in hadronic collisions are now available. Our results show that, for future precision measurements the full electroweak $\mathcal{O}(\alpha)$ corrections and probably also multiple photon radiation effects should be taken into account. The calculation of the non-resonant $\mathcal{O}(\alpha)$ corrections to $\mathrm{W}$ production has recently been completed [ 13] (see also Ref. [12]). As a first step towards a calculation of the $\mathcal{O}\left(\alpha^{2}\right)$ QED corrections, the effects of two-photon radiation in $\mathrm{W}$ and $\mathrm{Z}$ boson production at hadron colliders have been computed in Ref. [16].

\section{Acknowledgments}

We would like to thank the organizers of RADCOR-2002, for a delightful and inspiring conference experience. One of us (U.B.) is grateful to the Fermilab Theory Group, where part of this work was carried out, for its generous hospitality and for financial support. This research was supported in part by the National Science Foundation under grant No. PHY9970703.

\section{REFERENCES}

[1] D. Abbaneo et al. [LEPEWWG], arXiv:hepex/0112021.

[2] M. W. Grünewald, arXiv:hep-ex/0210003, to appear in the Proceedings of the "31st International Conference on High Energy Physics", Amsterdam, The Netherlands, July 2002.

[3] U. Baur, R. Clare, J. Erler, S. Heinemeyer, D. Wackeroth, G. Weiglein and D. R. Wood, in Proc. of the $A P S / D P F / D P B$ Summer Study on the Future of Particle Physics (Snowmass 2001) ed. N. Graf, arXiv:hepph/0111314.

[4] R. Brock et al., "Report of the working group on precision measurements", Proceedings of the Workshop on QCD and Weak Boson Physics in Run II, Fermilab-Pub-00/297, Fermilab, Batavia, Illinois, March - November 1999 (eds. U. Baur, R.K. Ellis and D. Zeppenfeld), p. 78, and hep-ex/0011009. 
[5] S. Haywood et al., "Electroweak physics", report of the Electroweak Physics Working Group of the 1999 CERN Workshop on SM physics (and more) at the LHC, CERN$\mathrm{TH} / 2000-102$ [hep-ph/0003275].

[6] S. Catani et al., "QCD", report of the QCD Working Group of the 1999 CERN Workshop on SM physics (and more) at the $\mathrm{LHC}$, CERN-TH/2000-102 hep-ph/0005025].

[7] U. Baur et al., "Report of the working group on photon and weak boson production", Proceedings of the Workshop on QCD and Weak Boson Physics in Run II, Fermilab-Pub00/297, Fermilab, Batavia, Illinois, March November 1999 (eds. U. Baur, R.K. Ellis and D. Zeppenfeld), p. 115, hep-ph/0005226.

[8] D. Wackeroth and W. Hollik, Phys. Rev. D55, (1997) 6788 hep-ph/9606398.

[9] U. Baur, S. Keller and D. Wackeroth, Phys. Rev. D59, (1999) 013002 hep-ph/9807417.

[10] U. Baur, S. Keller and W. K. Sakumoto, Phys. Rev. D57, (1998) 199 hep$\mathrm{ph} / 9707301$.

[11] U. Baur, O. Brein, W. Hollik, C. Schappacher and D. Wackeroth, Phys. Rev. D65, (2002) 033007.

[12] S. Dittmaier and M. Krämer, Phys. Rev. D65, (2002) 073007.

[13] U. Baur and D. Wackeroth, in preparation.

[14] S. Pozzorini, these proceedings.

[15] A. Kotwal et al. (The Tevatron Electroweak Working Group), Fermilab-FN-716; T. Affolder et al. (CDF Collaboration), Phys. Rev. Lett. 85, (2000) 3347; V. M. Abazov et al. (DØ Collaboration), Phys. Rev. D66, (2002) 032008. arXiv:hep-ex/0204009.

[16] U. Baur and T. Stelzer, Phys. Rev. D61, (2000) 073007.
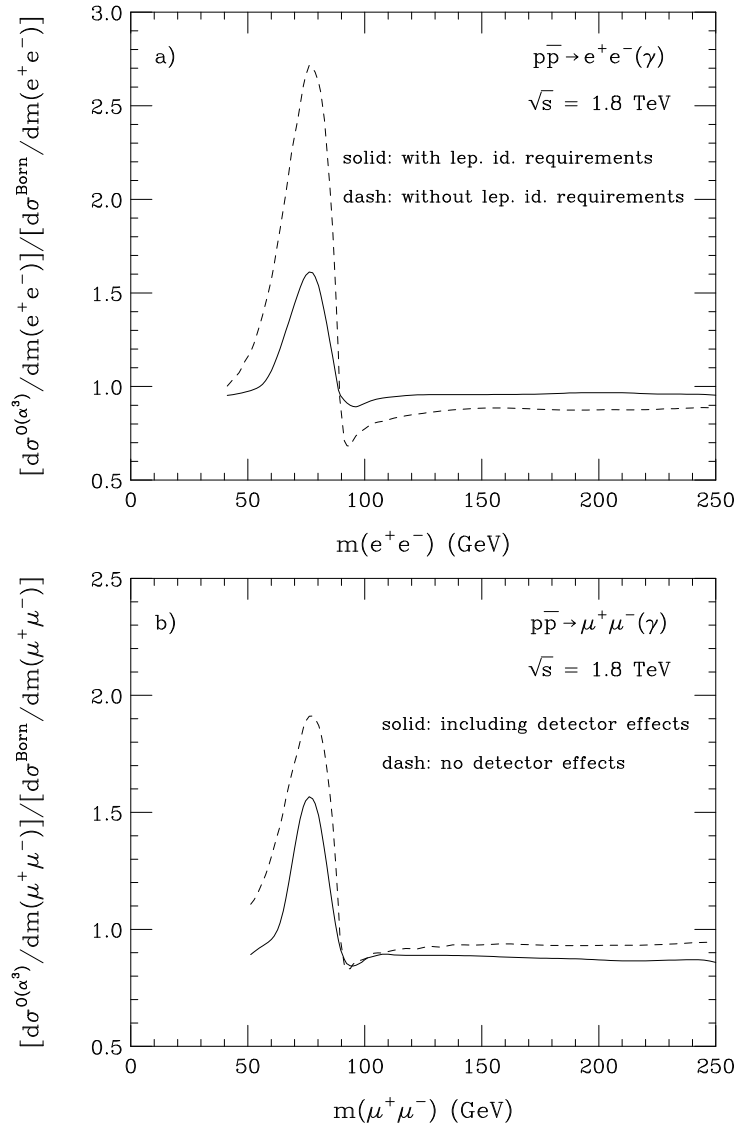

Figure 1. The relative corrections to the $m\left(e^{+} e^{-}\right)$ and $m\left(\mu^{+} \mu^{-}\right)$distributions in Drell-Yan production at the Tevatron due to the $\mathcal{O}(\alpha)$ QED corrections (from Ref. [10]). 

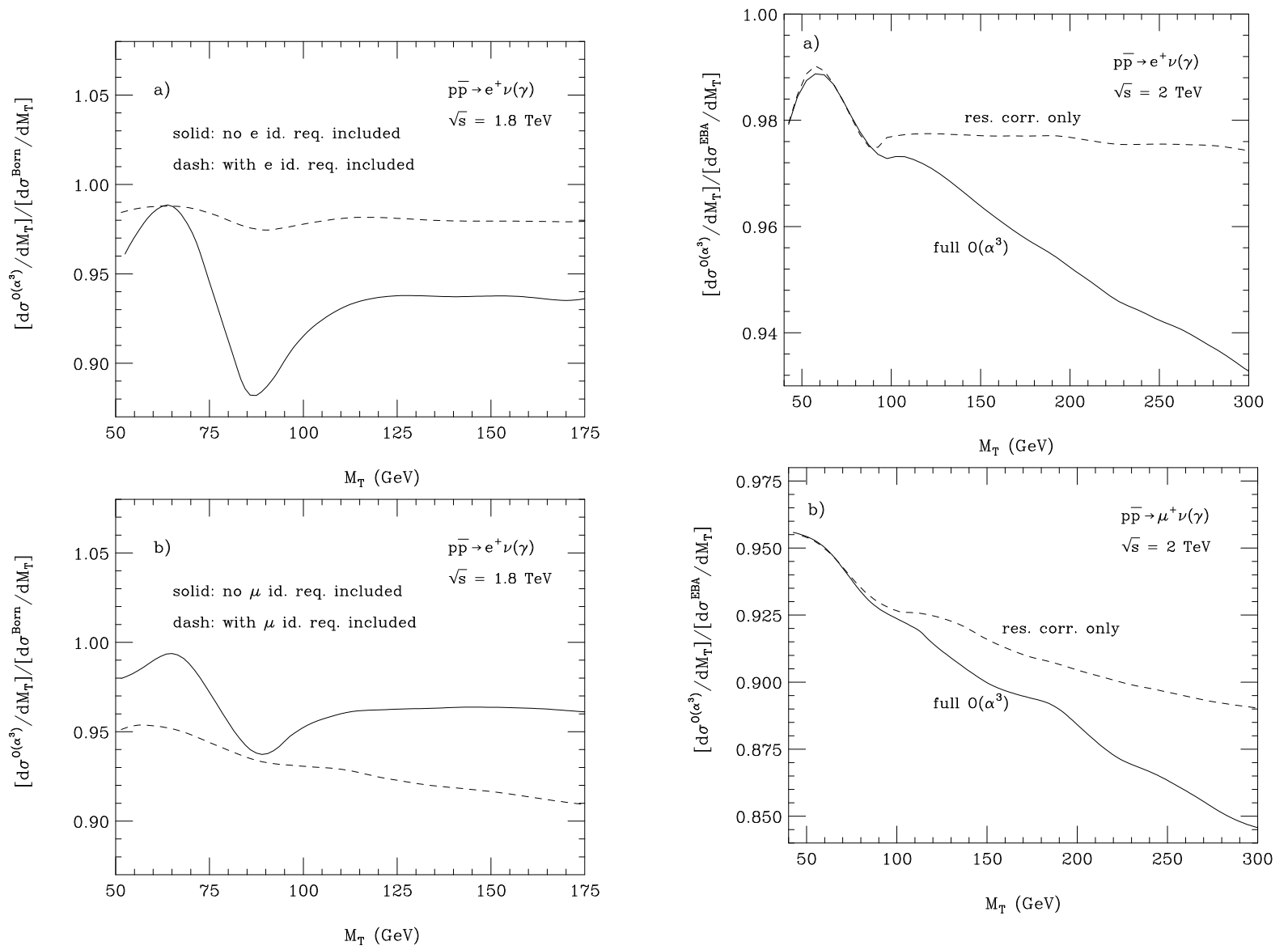

Figure 2. The relative corrections to the $M_{T}(l \nu)$ distributions at the Tevatron when taking into account electroweak $\mathcal{O}(\alpha)$ corrections (from Ref. [ 9).

Figure 3. The relative corrections to the transverse mass distribution at the Tevatron a) for electrons and b) for muons in the final state. The solid (dashed) line shows the result when the full (resonant) $\mathcal{O}(\alpha)$ electroweak corrections are taken into account. 


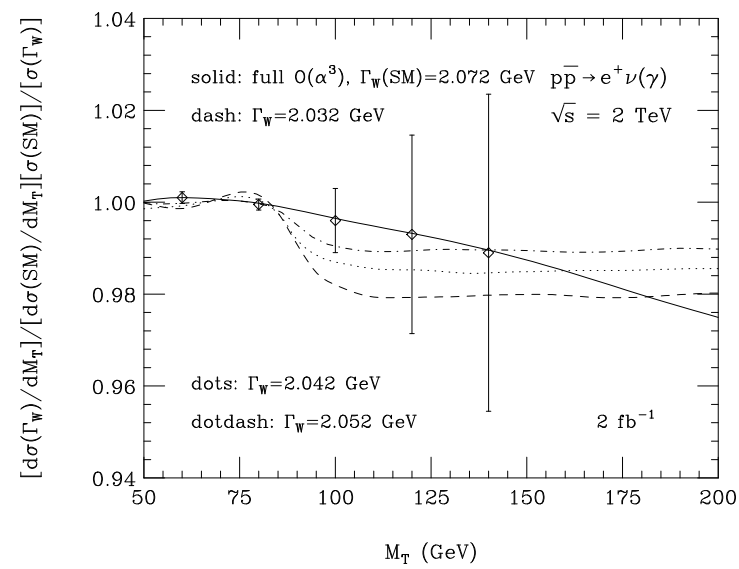

Figure 4. The ratio of the normalized transverse mass distribution to the normalized $M_{T}$ distribution with SM W width $\left(\Gamma_{W}(S M)=2.072 \mathrm{GeV}\right)$ for $p \bar{p} \rightarrow e^{+} \nu_{e}(\gamma)$ at the Tevatron when resonant $\mathcal{O}(\alpha)$ corrections only are taken into account. Similar results are obtained for muons in the final state. 